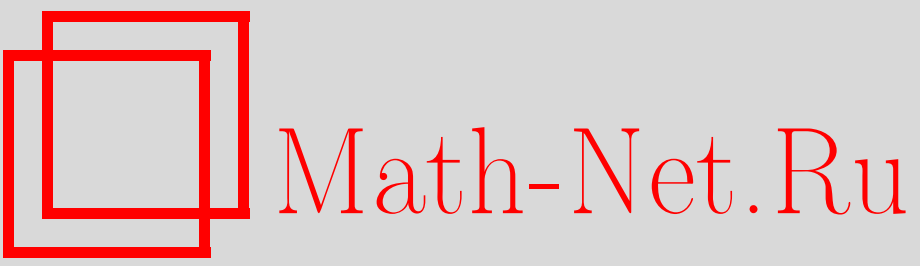

О. Р. Мусин, Проблема двадцати пяти сфер, УМН, 2003, том 58, выпуск 4, 153154

DOI: https://doi.org/10.4213/rm651

Использование Общероссийского математического портала Math-Net.Ru подразумевает, что вы прочитали и согласны с пользовательским соглашением http: //www . mathnet.ru/rus/agreement

Параметры загрузки:

IP: 18.234 .156 .22

26 апреля 2023 г., 17:42:13 


\title{
ПРОБЛЕМА ДВАДЦАТИ ПЯТИ СФЕР
}

\author{
О.Р. Мусин
}

1. Контактным числом $\tau_{n}$ называют наибольшее число непересекающихся и одинакового радиуса сфер в $\mathbb{R}^{n}$, которые одновременно касаются еще одной сфферы того же радиуса. Очень образно оно называется по английски - kissing number.

Очевидно, что $\tau_{2}=6$. Число $\tau_{3}$ явилось предметом знаменитой дискуссии 1694 г. между И. Ньютоном и Д. Грегори. Ньютон считал, что правильный ответ - 12, а его оппонент, что 13. Как следствие этой дискуссии в математике появилась проблема тринадцати сфер. Полное доказательство того, что $\tau_{3}=12$, появилось лишь в 1953 г. в работе ван дер Вардена и Шютте.

Легко показать, что $\tau_{4} \geqslant 24$. Рассмотрим сферы единичного радиуса с центрами в точках $( \pm \sqrt{2}, \pm \sqrt{2}, 0,0)$, где знаки и координаты можно переставлять всеми возможными способами. Все эти 24 сферы касаются единичной сферы с центром в начале координат.

В 1963 г. Кокстер, наряду с другими оценками для $\tau_{n}, n \leqslant 8$, получил оценку $\tau_{4} \leqslant 26$. (Доказательство Кокстера использовало одну гипотезу, которая была доказана Бёрёцки в 1978 г.) Значительный прорьв в проблеме верхней оценки для контактных чисел призошел в 1970-х гг. [1]. В 1979 г. независимо появились работы [2], [3], где с помощью метода Дельсарта было доказано, что $\tau_{8}=240$ и $\tau_{24}=196560$. Как оказалось, это единственные размерности, где этот метод дает точньй результат. В остальных случах (даже для $n=3$ ) верхняя оценка превьшает нижнюю. В работе [3] также было показано, что $\tau_{4} \leqslant 25$. Таким образом, взамен проблемы тринадщати сфер в размерности 3 появилась проблема двадцати пяти сфер в размерности 4.

Целью настоящей заметки является решение этой проблемы.

TeOpema. $\tau_{4}=24$.

2. Контактное число может быть определено по другому: $\tau_{n}$ - это наиболшшее число точек, которые можно расположить на единичной сфере $S^{n-1}$ в $\mathbb{R}^{n}$ так, чтобы угловое расстояние между любыми двумя из них было не меньше $60^{\circ}$.

Рассмотрим множество точек $M=\left\{p_{i}\right\}, i=1, \ldots, N$, на единичной сфере $S^{n-1}$ в $\mathbb{R}^{n}$. Пусть $\phi_{i j}-$ сферическое (угловое) расстояние между точками $p_{i}$ и $p_{j}$. Обозначим $t_{i j}=\cos \phi_{i j}=p_{i} p_{j}$, тогда $t_{i i}=1$.

Хорошо известно, что $\left(t_{i j}\right)$ - матрица Грама - является неотрицательно определенной. Шёнберг [4] обобщил этот факт и доказал, что матрица $\left(g_{i j}\right)$ также является неотрицательно определенной, где $g_{i j}=G_{k}^{(n)}\left(t_{i j}\right)$, a $G_{k}^{(n)}$ - ультрасферический многочлен (многочлен Гегенбауэра). Для $n=4 G_{k}^{(n)}(t)=U_{k}(t)=\frac{\sin (k+1) \phi}{(k+1) \sin \phi}, t=\cos \phi$, т.е. $U_{k}$ - нормированные $\left(U_{k}(1)=1\right)$ многочлены Чебышёва второго рода. Для неотрицательно определенной матрицы сумма ее элементов не меньше нуля, т.е. $\sum_{i, j} G_{k}^{(n)}\left(t_{i j}\right) \geqslant 0$. Если $f(t)=a_{0}+a_{1} G_{1}^{(n)}(t)+\cdots+a_{d} G_{d}^{(n)}(t)$, где все $a_{k} \geqslant 0, a_{0}>0$, то $F(M)=\sum_{i, j} f\left(t_{i j}\right) \geqslant a_{0} N^{2}$.

В нашем случае $t_{i j}=\cos \phi_{i j} \leqslant \cos 60^{\circ}=1 / 2, i \neq j$. Поэтому для $t_{i j}$ множество возможных значений $A=[-1,1 / 2] \cup\{1\}$. Пусть $A_{+}=\{t \in A: f(t)>0\}$. Тогда $F(M) \leqslant \sum_{t_{i j} \in A_{+}} f\left(t_{i j}\right)$. Правая часть этого неравенства содержит $N$ слагаемых $F_{i}=f(1)+f\left(t_{i j_{1}}\right)+\cdots+f\left(t_{i j_{m}}\right)$, $t_{i j_{k}} \in A_{+}$. Обозначим как $f_{\max }$ максимально возможную для таких $F_{i}$ величину. Тогда $F(M) \leqslant$ $f_{\max } N$. Получаем: $f_{\max } N \geqslant F(M) \geqslant a_{0} N^{2}$. Итак, доказано следуюшее

ПРЕДЛОЖЕНИЕ. $a_{0} N \leqslant f_{\max }$.

ЗАмечАниЕ 1. В работах [2], [3] это предложение применялось для $A_{+}=\{1\}$.

3. Зададим следующий многочлен 9-й степени:

$$
f(t)=53.76 t^{9}-107.52 t^{7}+70.56 t^{5}+16.384 t^{4}-9.832 t^{3}-4.128 t^{2}-0.434 t-0.016 .
$$

Разложение $f$ по $U_{k}=G_{k}^{(4)}$ имеет вид:

$$
f=U_{0}+2 U_{1}+6.12 U_{2}+3.484 U_{3}+5.12 U_{4}+1.05 U_{9} .
$$


Многочлен $f$ имеет на интервале $(-1,1)$ два корня: $t_{0}=-0.6079 \ldots$ и $1 / 2$. Если $t \in\left(t_{0}, 1 / 2\right)$, то $f(t)<0$ и функция $f(t)$ строго убьвает на интервале $\left(-1, t_{0}\right)$, т.е. $A_{+}=\left[-1, t_{0}\right) \cup\{1\}$.

Чтобы применить предложение для $n=4$ и $N=\tau_{4}$, необходимо оценить $f_{\max }$.

ЛЕмма. $f_{\max }=f(1)+2 f\left(\cos 150^{\circ}\right)=24.864396 \ldots$.

Из этой леммы сразу следует доказательство теоремы. Поскольку $a_{0}=1$, то из предложения вытекает $N \leqslant f_{\max }$, т.е. $24 \leqslant \tau_{4} \leqslant f_{\max }<25$, и $\tau_{4}=24$.

ЗАмЕчАниЕ 2 . Коэффициенты $a_{k}$ многочлена $f=\sum a_{k} U_{k}$ были найдены с помощью линейного программирования. Минимизировалась оценка на $f_{\max }$.

4. Доказательство леммы. Пусть на $\mathbb{S}^{3}$ задано множество точек $Q=p_{1}, \ldots, p_{m}, t_{i j} \leqslant$ $1 / 2$, если $i \neq j$. Для произвольной точки $p_{0} \in \mathbb{S}^{3}$ определена функция: $h\left(p_{0}, Q\right)=f(1)+f\left(t_{01}\right)+$ $\cdots+f\left(t_{0 m}\right)$, где $t_{0 k}=p_{0} p_{k}$. Для того чтобы оценить $f_{\max }$, требуется найти максимум функции $h(P), P=\left\{p_{0}\right\} \cup Q$, зависящей от $p_{0}, m$ и расположения множества $Q$ на сффере. Имеется дополнительное ограничение: $t_{0 k}<t_{0}$, которое означает, что расстояние $\phi_{k}$ от точки $p_{0}^{*}$ (антипод $p_{0}$ ) до точки $p_{k} \in Q$ меньше $\phi_{0}=52.5588^{\circ}, \cos \phi_{0}=-t_{0}$. (Здесь и далее показаны 4 знака после запятой.)

Так как $t_{0 k}=-\cos \phi_{k}$, то $h(P)=f(1)+f\left(-\cos \phi_{1}\right)+\cdots+f\left(-\cos \phi_{m}\right)$. Функция $f$ монотонно убывает на интервале $\left(-1, t_{0}\right)$, следовательно, при уменьшении $\phi_{k}$ величина $h(P)$ увеличивается. Тогда если $P$ - оптимальное множество, т.е. $h(P)=f_{\max }$, то ни одну из точек $Q$ нельзя сдвинуть по направлению к $p_{0}^{*}$. Для оптимального $P$ несложно доказать, что $Q$ - выпукльй, непривод имы й многогранник и $p_{0}^{*}$ лежит внутри $Q$. Здесь неприводимым называется такой многогранник на $\mathbb{S}^{d}$, что из любой его вершины выходит не менее $d$ ребер длины $60^{\circ}$.

Рассмотрим проекцию множества $Q$ на экватор из полюса $p_{0}^{*}$. Тогда минимальное расстояние у точек на проекции не меньше $\rho=78.0620^{\circ}$. Наименьшее из расстояний расположения 7 точек на $\mathbb{S}^{2}$ не превосходит $d_{7}=77.8695^{\circ}\left(\cos d_{7}=\operatorname{ctg} 40^{\circ} \operatorname{ctg} 80^{\circ}\right)$ [5]. Посколку $\rho>d_{7}$, то $m<7$.

Обозначим $h_{m}$ верхнюю грань значений $h(P)$ при фиксированном $m$ и $Q^{(m)}-$ соответствующее $Q$ для оптимального $P$, тогда $f_{\max }=\max \left\{h_{m}\right\}, 1 \leqslant m \leqslant 6$. Исходя из свойств оптимального $Q$, оценим $h_{m}$. Очевидно, что $h_{1}=f(1)+f(-1)=24.48 . Q^{(2)}-$ дуга $p_{1} p_{2}$ длины $60^{\circ}$. Наибольшее значение $h(P)$ достигается, когда $p_{0}^{*}$ лежит в середине $p_{1} p_{2}$, т.е. $h_{2}=f(1)+$ $2 f\left(\cos 150^{\circ}\right)=24.8644 . Q^{(3)}$-правильньй треугольник, а $Q^{(4)}$-правилнный тетраэдр со сторонами $60^{\circ}$. Вычисления показывают, что $h_{3}=24.8345<h_{2}, h_{4}=24.8180<h_{2}$. У $Q^{(5)}$ имеется вершина, из которой выходит 4 ребра длины $60^{\circ}$. Обозначим через $\alpha$ длину наименьшего ребра, лежащего против этой вершины, тогда $60^{\circ} \leqslant \alpha \leqslant 90^{\circ}$. Численный анализ показывает, что наиболшшее значение $h(P)=24.6856$ достигается при $\alpha=60^{\circ}$, т.е. $h_{5}<h_{2}$. Для $m=6$ верхняя грань $h(P)$ достигается, когда $Q$ - октаэдр. Вычисления показывают, что $h_{6}=22.5205<h_{2}$. Таким образом, все $h_{m} \leqslant h_{2}$ и лемма доказана.

Автор благодарит И. А. Дынникова за рецензирование работы.

\section{СПИСОК ЛИТЕРАТУРЫ}

[1] Дж. Конвей, Н. Слоэн. Упаковки шаров, решетки и группы. М.: Мир, 1990. [2] В.И. Левенштейн // Докл. АН СССР. 1979. Т. 245. С. 1299-1303. [3] А. М. Odlyzko, N. J. A. Sloane // J. Combin. Theory Ser. A. 1979. V. 26. № 2. P. 210-214. [4] I. J. Schoenberg // Duke Math. J. 1942. V. 9. P. 96-108. [5] K. Schütte, B. L. van der Waerden // Math. Ann. 1951. V. 123. P. 96-124.

Московский государственньй

Принято редколлегией университет им. М. В. Ломоносова 14.05 .2003 